\title{
Mathematical Modelling and Simulation of the Bipodal Gait
}

\author{
Xue M. González ${ }^{1}$, Diego F. Valero ${ }^{1}$, Oscar Avilés ${ }^{1}$, Ruben D. Hernández ${ }^{2, *}$ and Jair L. Loaiza ${ }^{3}$ \\ ${ }^{1}$ Department of Mechatronics Engineering, Innovatic Research Group, Piloto de Colombia University, Colombia. \\ ${ }^{2}$ Department of Biomedical Engineering, Davinci Research Group, Nueva Granada Military University, Colombia. \\ ${ }^{3}$ Department of Mechanical Engineering, University of Campinas, Brasil.
}

Received 2 June 2019; Accepted 31 January 2020

\begin{abstract}
In this article we present a mathematical model of the bipedal gait as well as the respective simulation of the joint movement that makes it possible. The data acquisition was carried out with the Tracker ${ }^{\circledR}$ software, and the dynamic simulation is

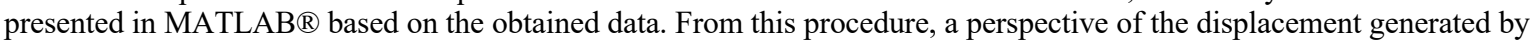
the lower train of the digitized human body is generated, which allows a study of the most concrete and successful movement for medical studies, since it allows the implementation of specialized designs and that meet specific characteristics in structures to reproduce the movement of the different joints.
\end{abstract}

Keywords: Mechanical motion, biomechanical, biomechatronics.

\section{Introduction}

The reproduction of the displacement generated by the inferior train of the human body is a key piece for the design and implementation of medical prothesis. The previous statement is one of the main reasons to develop this project since that by obtaining specialized simulations due everyone, allows the creation of structures with better adaptation to the characteristics of every single person than generic structures. This work allows to make a study of the movement that could be applied to physical projects to contribute to the development and implementation of structures capable of mimic the human gait. This phenomenon of movement has been widely studied in the literature and keeping in mind that, here is introduced one simulation method based on the tracking of the joints during the gait through the software Tracker ${ }^{\circledR}$ and then, with the data obtained it is carried out the specific dynamic simulation in MATLAB $R$.

The study of motion beyond the social ambit is an essential part of this project thinking in the engineering and medical applications in order to generate useful advancements in technology for the people with some sort of difficulties that require prothesis and not only for the inferior train since that the method can be extrapolated to track the motion of whatever part a dynamic process.

In the literature there are researches that present working approximations of mathematical models and simulations of the human gait to a prototype due posterior implementation. To know, the inferior extremities of human beings (legs and feet) are taken as a study object because it allows the development of working structures that consider the movement generated in the duty cycle of the gait.

The functions that describe the parameters for the walk first appear with a close relation of the segmentation of the extremity for the designing structure. To make the interpretation of such relations it has have been used software

*E-mail address: ruben.hernandez@unimilitar.edu.co ISSN: $1791-2377$ @ 2020 School of Science, IHU. All rights reserved. doi:10.25103/iestr.131.03

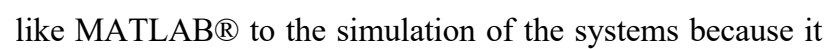
allows the visualization of variables like torques to get more stable structures, such that their parameters give response to the anthropometrical measures that guarantee that the models are going to fit to the individual standards.

The previous has had an impact in various fields of medicine, and its analysis has made it possible to improve the motion patterns through designs that lies on the segmentation of the extremity. Specifically, in [8] appears a prototype of an exoskeleton designed for a person and which main purpose was to analyze the response of the structure towards the reactions caused by the motion, and just like that been able to establish restrictions for future projects.

Currently, some of the models present significant weaknesses on the dynamic tracking due to data of the walk variables which might require very specific tracking techniques in order to satisfy the design requirements. Because of this it is sought to increase the tools of data acquisition that in general could be extrapolated to application of dynamic structures analysis.

\section{Methods and Materials}

A key concept to understand the work that is been developed in this document is the sequences of the gait. That is to say, the episodes of the displacement generated between the beginning of the movement and the phase right before the redoing of the sequence. In other words, the gait is a repetition of sequences in which every one of the inferior extremities go through by a stance phase (where the foot is touching the floor), a transitional phase also called the oscillation phase (where the foot leaves the floor and move forward to another contact point) and finally the arrival of the foot to that new contact point with the floor.

The oscillatory process is giving since the forefoot zone take off until the next contact with the floor. In association with the gait time, the step process is normally constituted by the initial inertial velocity of the subject that usually takes an 
$60 \%$ of the sequence and the oscillation takes the remaining $40 \%$.

The same thing happens with the collateral limb which is displaced a $50 \%$ of the time, that shows an existence of two bipodal processes (Fig. 1) each with a duration of $10 \%$. The normal extension of this processes depends directly on the velocity: the more oscillations forward to the foot support increases the velocity [1].

The time where only one foot is in contact with the floor and the collateral limb is in the oscillation process is called monopodal support period. The duration of the left monopodal support in regular conditions matches with the right oscillation and vice versa. So, the duration of the foot in one series is equivalent to the sum of the monopodal support time with respect to the two support times. The distance between the respective foot supports is called the stride length [1].

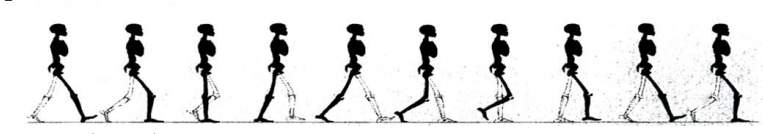

Fig. 1. Gait cycle [1].

Following that, in the human gait takes place two forms of energy exchange: kinetical and potential energy, and the energy transfer amongst the segments. This means that during the bipodal support process, the center of gravity is in its lower position and therefore it presents its maximum speed.

Taking into account the energy, at that point the potential energy is in its lowest spot and thus the kinetical energy is on its highest spot 3-27. In the half-process of the monopodal support the trunk rises on top of the leg that provides the support slowing down and turning kinetical energy into potential energy. Due to the rest of the monopodal support, the trunk goes down to its original height increasing its velocity [1].

The development of the gait in the series is divided into various sequentially constituents. In general conditions is it possible to find the next sequential events:

- Contact of the hindfoot with the floor

- Full contact of the foot's sole

- Takeoff of the hindfoot

- Takeoff of the forefoot

- Oscillation time of the extremity

- Next hindfoot contact with the floor. cycle:

It is possible to establish the next sub-phases of the gait

Load reception phase: between the initial support contact of the foot from the hindfoot to the forefoot.

- Middle phase of the support: it goes until the moment of takeoff of the hindfoot.

- Takeoff phase: until the moment in which the forefoot goes off the floor.

Another sub-phase considers the support made up by five fundamental periods, representing the gait cycle with percentages:

\section{a. Support phase}

Initial contact (IC) $0.2 \%$ : It represents the take of contact of the foot with the floor. Even that this phase has non welldefined limits, this leads to a clear goal: the positioning of the limb to start the support phase is through the contact with the hindfoot with the floor.
Initial support phase or response to the load (IS) $0-10 \%$ : it takes place between the initial contact and takeoff of the forefoot of the contralateral limb in regular conditions. In this phase the inferior extremity must absorb the initial impact keeping the stability of both support and progression. During this period the knee gets bent and the heel makes a plantar flexion controlled by the quadriceps and the tibialis anterior; at the same time the hips gets stabilized.

Middle support phase (MS) $10-30 \%$ : is the progression of the body over the steady foot keeping stability over the extremity and the trunk.

Initial support phase (ISP) $30-50 \%$ : it starts with the takeoff of the forefoot and ends it up when the contralateral limb gets in contact with the floor. At this point the upper body goes forward the steady foot position, falling ahead.

Pre-oscillating phase (PO) $50-60 \%$ : The initial contact of the contralateral limb indicates the end of the current phase; it ends it up with the takeoff of the forefoot. The main function of the limb is the preparations to perform the oscillation facilitated by the entrance of the load to the collateral limb towards which the load is transferred [1].

\section{b. Oscillation phase}

Initial oscillation phase (IO) $60-73 \%$ : it corresponds approximately to the first third of the oscillation period. Its bounded by the takeoff of the limb and the moment where this reaches the contralateral limb. The limb goes forward through a hip flexion to insurance the proper distance between the foot and the floor.

Middle oscillation phase (MO) $73-87 \%$ : it begins in the cross path of thee limbs, and it end it up when the oscillating tibia reaches a vertical position once it overpasses the support limb.

Final oscillation phase (FO) $87-100 \%$ : bounded by the next contact of the oscillating limb with the floor allowing a new stride. At this point it is necessary finalize the advancement of the limb and perform the preparation to the upcoming contact. A braking action of the hip and knee flexion is produced [1].

Functional interpretation and impact cushioning: the impact cushioning, the stability maintenance and advance, shape the motion and muscle control on the foot which performs the segment roll between the extremity and the floor. One of the main functions of the structures of the foot is the attenuation of the impact on each step.

\section{c. Contact phase}

Hindfoot support: is starts the floor contact. The speed rate of approximation is relatively high due the general motion rate. Foot sole support: with the entrance contact of the forefoot with the floor, it starts the full support on the foot.

Forefoot support: The takeoff of the heel, stablish the change of the sore foot support to the forefoot [1].

\section{Kinematic Model of the Walk}

It is the joint between to long bones: the femoral bone and the tibial bone; those bones are the major constituents of the inferior limb. The mobility and stability of the knee are essential in the normal gait pattern. During the support process the knee is the main stability agent of the limb; likewise, in the oscillation process the knee flexibility is the most important factor to enable the advancement of the limb [1]. 
Kinematics of the knee: The knee is a joint characterized by a widely range of motion in the sagittal plane, and small arches of frontal and transversal mobility. The flex-extension motion in the sagittal plane is employed for the progression in the support phase and to the advance of the limb in the oscillation, meanwhile the frontal plane motion facilitates the vertical equilibrium on the limb during the monopodal support phase. The transversal rotation is produced in consonance with the anterior limbs. In the sagittal plane the knee makes an alternant motion of flexion and extension, introducing four trajectories of the motion in every stride. The normal knee motion during the gait goes between $0^{\circ}$ and $70^{\circ}$ [1].

The intervals between flexion and extension of the gait cycle (GC) are shown in the Table 1.

Table 1. Knee motion arches in a stride [1].

\begin{tabular}{c|c}
\hline Movement & Interval Gait Cycle \\
\hline Flexion until $18^{\circ}$ & $0-15 \%$ \\
Extension until 5 & $15-40 \%$ \\
Flexion until 65 & $40-70 \%$ \\
Extension until $2^{\circ}$ & $70-97 \%$ \\
\hline
\end{tabular}

In the frontal plane along every gait sequence, the knee is exposed to abduction as well as adduction [13]. The introduced phases in the knee motion are:

- Final oscillation phase (FO): As a preparation of the limb for the support, the knee must go from the needed flexion in the oscillation to an extension stance. The quadriceps lift the lower extremity while the thigh is keeping ahead by around $30^{\circ}$.

- Initial contact phase (IC): Whenever the foot touches the ground the knee is in an extension form: $2^{\circ}$ to $5^{\circ}$ of flexion.

- Initial support phase or response to the load (IS): The knee is bent by $15^{\circ}$.

- Middle support phase (MS): Extension of the knee with the stability function in the support.

- Final support phase (FS): The knee extension is completed, and it maintains the stability in the support and advancement of the step [15].

- Pre-oscillation phase (PO): It is produced a knee flexion and it prepares for the oscillation movement.

- Middle oscillation phase (MO): It is generated a passive extension on the knee that allows the advance of the limb [1].

Hip joint: The hip is the junction point between the locomotion system and the system body. The kinematics of the joint movement is defined as the trajectory of the thigh related to the vertical [17].

Table 2. Movement arches of the hip and thigh during the stride [1].

\begin{tabular}{c|c|c|c}
\hline Movement & Thigh & Hip & Instant \\
\hline Flexion & $20^{\circ}$ & $30^{\circ}$ & $0 \%$ \\
Extension & $20^{\circ}$ & $10^{\circ}$ & $50 \%$ \\
Flexion & $25^{\circ}$ & $35^{\circ}$ & $85 \%$ \\
\hline
\end{tabular}

The stabilization of the trunk mass over the hip introduces a strong requirement of muscle control in the support period. Nevertheless, there exists a mechanism to save energy that consist in the replacement of the muscular action by passive forces once the load of the limb phase is over [1]. The movement phases in the hip are:

- Initial contact phase (IC): The hip takes a stand of $30^{\circ}$ flexion.

- Initial support phase or load response (IS): Its function is the maintenance of the position in the sagittal and frontal planes.

- Middle oscillation phase (MO): Is the continuation of the hip-flexion: $10^{\circ}$.

- Final oscillation phase (FO): Cessation of the hipflexion [1].

Ankle joint:_As far as these joint concerns, the freedom degrees based on the position are:

Table 3. Ankle movement arches in a stride [1].

\begin{tabular}{c|c}
\hline Movement & Interval \\
\hline Flexion plant until $7^{\circ}$ & $0-7 \% \mathrm{MC}$ \\
Flexion dorsal until $10^{\circ}$ & $7-48 \% \mathrm{MC}$ \\
Flexion plant until $20^{\circ}$ & $48-62 \% \mathrm{MC}$ \\
Flexion dorsal until $0^{\circ}$ & $62-100 \% \mathrm{MC}$ \\
\hline
\end{tabular}

Next it is analyzed the kinematics of the leg motion, In the Fig. 2 are shown the links to keep into account due the analysis of the motion angles of each articulation.
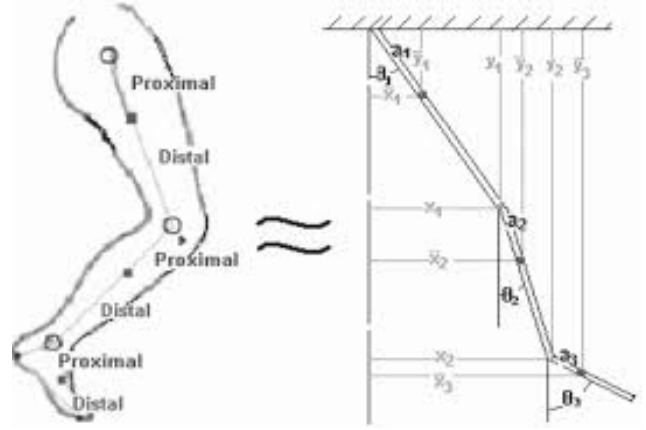

Fig. 2. One leg model [2].

There is another way to analyze the motion of the bipedal gait: the seven-links model that allows to perform the movement regarding to both legs and the trunk for higher accuracy.

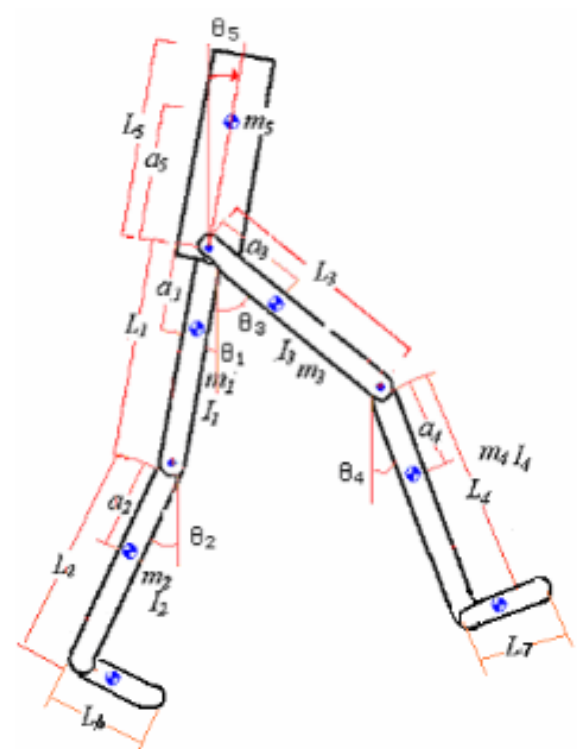

Fig. 3. Seven-links model [2]. 
In order to obtain the equation of the analyzed system, it is first formulated the Lagrange function (L) also known as the kinetic potential or lagrangian of the system, and is defined by:

$L=T-V$

Being $\mathrm{T}$ the kinetic energy and $\mathrm{V}$ the potential energy of the system. The lagrangian gives the amount of transported energy from one form to the other, respectively. Now, having into account the inverted pendulum model:
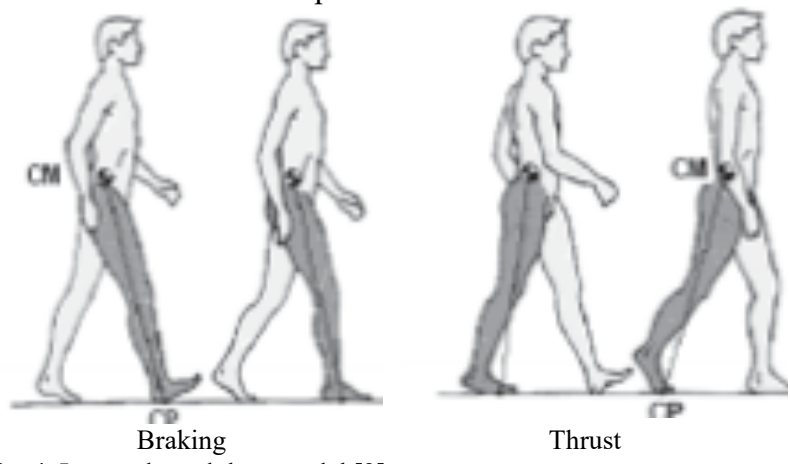

Fig. 4. Inverted pendulum model [2].

From the lagrangian dynamics it is possible to determine the movement equation or Euler-Lagrange equation. In order to do so, the lagrangian is derived due the vector generalized coordinates regardless external torques:

$\frac{d}{d t}\left(\frac{\partial L}{\partial \dot{q}}\right)-\left(\frac{\partial L}{\partial q}\right)=0$

Coordinate vector [2].

$\frac{d}{d t}\left(\frac{\partial L}{\partial \dot{\theta}}\right)=m l^{2} \ddot{\theta}$

Acceleration, position and speed [2]. The mathematical model for the inverted pendulum is defined as follows:

$\ddot{\theta}=\frac{g}{l} \sin \theta$

where $\theta$ is the position, (2) is the derivative of the speed, (3) is the derivative of the acceleration, $m$ is the mass, $g$ is the gravitational constant and 1 is the length.

The simulation determines the requirements to consider in the gait process. These requirements could be the position of each joint to effect one individual angular position for further analysis.

In [3] is possible notice that the planification of some particular walk was implemented with the trajectories generation method, which has an indirect impact on the joints because it is worked with the position and orientation of the final effectors, from that it is possible to notice the requirement of the inverted kinematics resolution.

Regarding to the systems controller based on the generation trajectories method is implemented the closed loop with the proportional derivative PD, which shows optimal results accordingly with the literature.
The optimal control selection is a substantial matter to obtain the desired behavior of the system. To do so, there must be first stablished the parametric characteristics of the design. These parameters are: the length and mass of the segments as well as specific characteristics of the person.

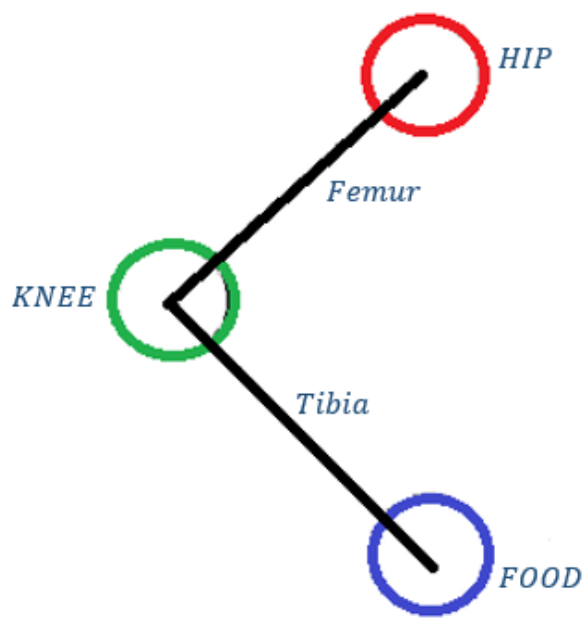

Fig. 5. Simplified parts of one leg [3].

In [3] are presented the simulation results of the walk of people around 20 years old, $1,65 \mathrm{~m}$ high and $70 \mathrm{~kg}$; the study showed a generalized gait pattern in controlled conditions. This allows to establish general patterns for the prothesis design based on statistical data analysis.

In such study it has been considered every segment as a solid cylinder, and to do that it was first necessary to establish the optimal diameter of each segment. In the Table 4 appear the measurements and body mass of every single portion of the structure [3].

Table 4. Measurements and mass of the leg parts [3].

\begin{tabular}{c|c|c|c|c}
\hline Part & $\begin{array}{c}\text { Mass } \\
(\mathbf{G})\end{array}$ & $\begin{array}{c}\text { Length } \\
(\mathbf{C m})\end{array}$ & $\begin{array}{c}\text { Radius } \\
(\mathbf{C m})\end{array}$ & $\begin{array}{c}\text { Density } \\
\left(\mathbf{G} / \mathbf{C m}^{\wedge} \mathbf{3}\right)\end{array}$ \\
\hline Hip & 4000 & 35 & 7 & 0.866 \\
Thigh & 7004 & 40 & 6 & 1.542 \\
Calf & 2924 & 40 & 5 & 0.646 \\
Foot & 1020 & 14 & 8 & 0.644 \\
\hline
\end{tabular}

In other cases, the inverse kinematics is used. The position of the hip has been taken as the origin point of the thigh and calf vectors. These vectors have an invariable radius and length. As an example, $0.4 \mathrm{~m}$ of each is taken, so that the maximum radius that the leg can reach is $0.8 \mathrm{~cm}$. To simplify the computation of the results it is important that the length of the thigh and calf to be the same, as shown later.

The location of the ankle was made by means of a Cartesian system of coordinates $(\mathrm{x}, \mathrm{y})$ in relation to the hip. With these data, the equivalence of the distances is obtained by means of the analysis by means of a right triangle.

Defined in polar coordinates the point where wants to put the ankle, the angle formed between the thigh and the hip is calculated so that the foot points just at that position. Since the thigh and the calf have an exactly equal length, the point where they converge will always fall in the vertical of half the distance between hip and foot, and the knee will always be in that line.

It is observed that for the points of hip, knee and the midpoint between hip and ankle, a right triangle is formed as in the previous case, only that now the hypotenuse is the thigh 
itself and its length is known, so that only the described angle of the thigh must be calculated with respect to the $\mathrm{x}$-axis. To calculate the angle of the femur with respect to the vertical (remember that it is half the length between hip and ankle), the hypotenuse (thigh) [3].

Table 5. Measurements and masses of the parts of the legs. [3].

\begin{tabular}{c|c|c|c|c}
\hline Time & $\begin{array}{c}\text { Left } \\
\text { Hip }\end{array}$ & $\begin{array}{c}\text { Left } \\
\text { Knee }\end{array}$ & $\begin{array}{c}\text { Right } \\
\text { Hip }\end{array}$ & $\begin{array}{c}\text { Right } \\
\text { Knee }\end{array}$ \\
\hline 0 & 5,186 & 0 & 14,911 & $-47,952$ \\
0,033 & 3,729 & 0 & 19,727 & $-44,065$ \\
0,066 & 2,156 & 0 & 22,659 & $-41,503$ \\
0,1 & 0,489 & 0 & 23,864 & $-13,736$ \\
\hline
\end{tabular}

The generated signal for the angular position in matrix form is obtained through an interpolation once the previous data is obtained. Then, that signal is represented by a simulation with MathModelica ${ }^{\circledR}$ as a reference signal using the TimeTable ${ }^{\circledR}$ block.

The time points and values of the function are stored in a matrix table $[i, j]$, where the first column contains the time points and the second column the data to be interpolated.

At this point, the objective of knowing the angle that the extremities of a supposed leg must reach a specific point has already been achieved [4]. From this, the data obtained in Table 1 (length of the parts) [3] are taken and the movements of the extremities (mainly) are described, including the leg, which is the limb involved in human displacement (bipedal gait). The links or analysis points on the leg are shown in fig 6.

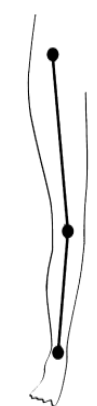

(a)

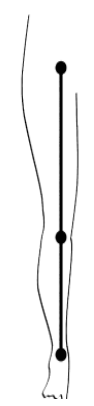

(b)
Fig. 6. a) Orthopedic torsion, b) Orthopedically correct leg [4].

It is well known that the leg is made up of bones which provide structural support; the muscles, which are responsible for generating power and tendons that allow the coverage of joints and help the movement. In the production of the movement, loads such as torsion, compression effects among others, mainly affect the bones. And due to the correct position of each of these components, the movement is generated together to generate a uniform displacement. The human gait is produced by means of two legs, which have the same characteristics with a main joint in the hip that allows the balance for the alternating movement of the displacement [4]. With the definition of the series of the gait, the parameters of the movement for the mathematical analysis are generated. Let L1 be the instant in which the swing leg is about to touch the ground (the impact between the rotating foot and the ground), the other foot starts its movement to execute half of the plane process of this phase. That is, the speed jump of the current leg (the leg that is not in support), is due to the impact of the other leg with the ground. [6]

The parameter chosen to evaluate mechanical effectiveness is "mechanical power of the joint" which is the product of the angular velocity of a joint and the net moment of the joint. It is said that the positive power of the joint occurs when the sign of the moment in a given plane matches with the sign of the angular velocity or the direction of the change in the angle of the joint; while the negative power, which is the absorption of energy, occurs when the signs do not match [7].

Given the model segments are rigid, it was necessary to include deformable "spring" elements in the joints (to excessively reduce sharp force changes, and thus stabilize the models). The above, in addition to the resultant ground reaction force curves, depending on the values of stiffness and damping, allow an analysis in the sagittal and vertical plane for the force generated with respect to the degrees of freedom in the coordinate system (X and $\mathrm{Z}$ ). The highest stiffness settings progressively approximate stiffness and produce sagittal floor reaction force curves that oscillate sharply from acceleration to deceleration, a maximum of three acceleration peaks and three deceleration peaks that occur when hip stiffness It is set to $50,000 \mathrm{lbf}$.in/degree. The closest approximation to the typical curves of the real sagittal occurred with the hip stiffness established at 1500 lbf.in/degree. Based on the information obtained, it is determined that the damping has a greater effect on the vertical reaction curves of the floor, since it produces more realistic movement curves, and in turn, the cushioning adjustments in the ankle joint were more significant than in the knee or the hip.

This is evident in the simulations, when considering the oscillatory curves in the movement of the gait, where the ankle presents a change of position with respect to the orthogonal axis to the ground which is greater than in any of the other joints. The general model takes into account the previously defined parameters and manages to predict the forces in the system with good accuracy by using proportions of mass and inertial properties in each segment. This is achieved through kinematic functions for each joint, derived from a single gait cycle. The results of the verification exercises were judged satisfactory, since they predicted the time and approximate magnitude of every single of the main power fluctuations in the three joints [7].

\section{Results}

For the mathematical modeling, the direct kinematics method with the vector technique is employed. In the first instance, by the segmentation of the leg into three (3) parts:

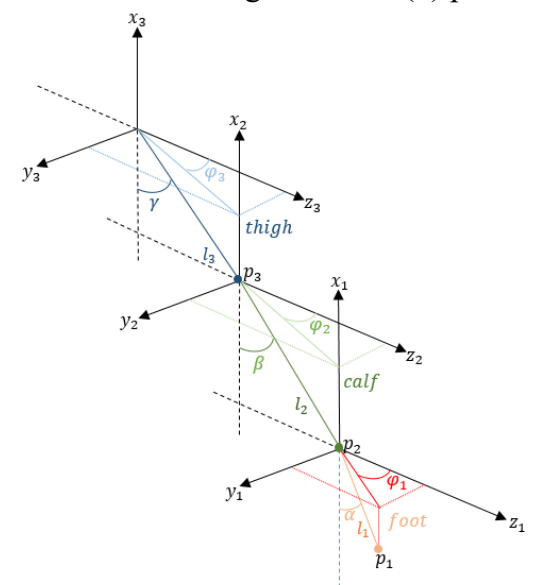

Fig. 7. Movement frames, angles and moments. 
Xue M. González, Diego F. Valero, Oscar Avilés, Ruben D. Hernández and Jair L. Loaiza/

Journal of Engineering Science and Technology Review 13 (1) (2020) 18 - 25

We proceed to determine the position, velocity and acceleration of a points $P$, with respect to each of the 3 links. For this, the values of average measures of the population for mathematical modeling are considered. Due the dynamic variation of the value of the angles, these are expressed as a time variable function. Such angles and combination of angles of the segments are those that allow the movement of the leg and then the simulation of one or several steps consecutively. $\mathrm{P}$ Position, for modeling the position of points $\mathrm{P}$ :

$p_{1}=-l_{1} \cos \alpha \widehat{l_{1}}+l_{1} \sin \alpha \cos \varphi_{1} \widehat{\jmath_{1}}+l_{1} \sin \alpha \sin \varphi_{1} \widehat{k_{1}}$

$p_{2}=-l_{2} \cos \beta \hat{l}_{2}+l_{2} \sin \beta \cos \varphi_{2} \widehat{J_{2}}+l_{1} \sin \beta \sin \varphi_{2} \widehat{k_{2}}$

$p_{3}=-l_{3} \cos \gamma \widehat{\imath_{3}}+l_{3} \sin \gamma \cos \varphi_{3} \hat{\jmath}_{3}+l_{3} \sin \gamma \sin \varphi_{3} \hat{k}_{3}$

According to Figure 8, speeds and accelerations can be calculated by taking the position vector $\overrightarrow{r_{1}}$ that relates the tip of the food to the coordinate system $\left(x_{1}, y_{1}, z_{1}\right)$, the position vector $\overrightarrow{r_{2}}$ taken from the tip of the food with respect to the coordinate system $\left(x_{2}, y_{2}, z_{2}\right)$ where:

$\overrightarrow{r_{2}}=l_{2}+l_{1} \quad o \quad \overrightarrow{r_{2}}=l_{2}+\overrightarrow{r_{1}}$

Likewise, the vector $\overrightarrow{r_{3}}$ relates the position tip of the food to the coordinate system $\left(x_{3}, y_{3}, z_{3}\right)$.

$\overrightarrow{r_{3}}=l_{1}+l_{2}+l_{3} \quad o \quad \overrightarrow{r_{3}}=l_{3}+\overrightarrow{r_{2}}$

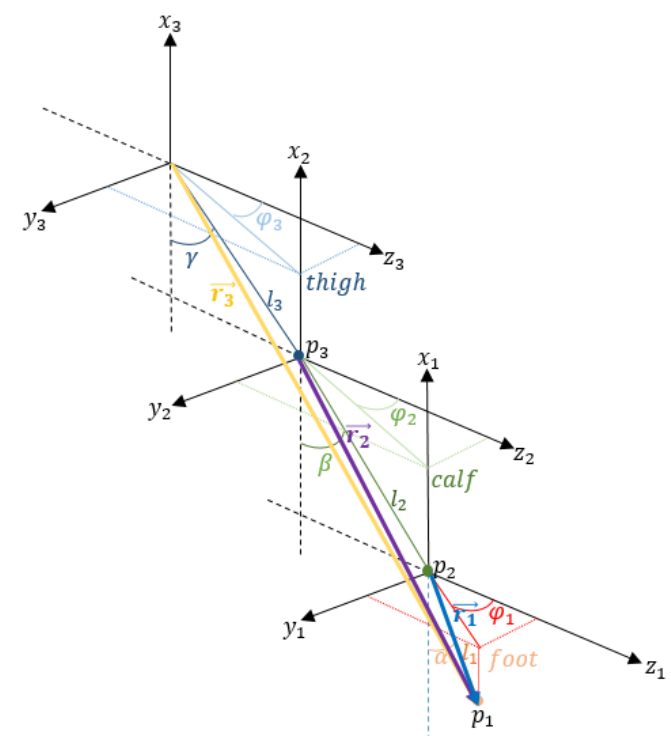

Fig. 8. Velocity and acceleration analysis diagram.

The modeling of the velocity of point $\mathrm{P}_{1}$ :

$$
\begin{aligned}
\overrightarrow{r_{1}}=\left(l_{1} \dot{\alpha} \sin \alpha\right) \hat{\imath} & +\left(l_{1} \dot{\varphi_{1}} \sin \alpha \cos \varphi_{1}+l_{1} \dot{\alpha} \cos \alpha \sin \varphi_{1}\right) \hat{\jmath} \\
& +\left(-l_{1} \dot{\varphi_{1}} \sin \alpha \sin \varphi_{1}\right. \\
& \left.+l_{1} \dot{\alpha} \cos \alpha \sin \varphi_{1}\right) \hat{k} \\
\overrightarrow{r_{2}}=\left(l_{1} \dot{\alpha} \sin \alpha+\right. & \left.l_{2} \dot{\beta} \sin \beta\right) \hat{\imath} \\
& +\left(l_{1} \dot{\varphi}_{1} \sin \alpha \cos \varphi_{1}+l_{1} \dot{\alpha} \cos \alpha \sin \varphi_{1}\right. \\
& \left.+l_{2} \dot{\varphi}_{2} \sin \beta \cos \varphi_{2}+l_{2} \dot{\beta} \cos \beta \sin \varphi_{2}\right) \hat{\jmath} \\
& +\left(-l_{1} \dot{\varphi_{1}} \sin \alpha \sin \varphi_{1}+l_{1} \dot{\alpha} \cos \alpha \sin \varphi_{1}\right. \\
& \left.-l_{2} \dot{\varphi}_{2} \sin \beta \sin \varphi_{2}+l_{2} \dot{\beta} \cos \beta \sin \varphi_{2}\right) \hat{k}
\end{aligned}
$$

$$
\begin{aligned}
\overrightarrow{r_{3}}=\left(l_{1} \dot{\alpha} \sin \alpha+\right. & \left.l_{2} \dot{\beta} \sin \beta+l_{3} \dot{\gamma} \sin \gamma\right) \hat{\imath} \\
& +\left(l_{1} \dot{\varphi}_{1} \sin \alpha \cos \varphi_{1}+l_{1} \dot{\alpha} \cos \alpha \sin \varphi_{1}\right. \\
& +l_{2} \dot{\varphi}_{2} \sin \beta \cos \varphi_{2}+l_{2} \dot{\beta} \cos \beta \sin \varphi_{2} \\
& \left.+l_{3} \dot{\varphi_{3}} \sin \gamma \cos \varphi_{3}+l_{3} \dot{\gamma} \cos \gamma \sin \varphi_{3}\right) \hat{\jmath} \\
& +\left(-l_{1} \dot{\varphi_{1}} \sin \alpha \sin \varphi_{1}+l_{1} \dot{\alpha} \cos \alpha \sin \varphi_{1}\right. \\
& -l_{2} \dot{\varphi_{2}} \sin \beta \sin \varphi_{2}+l_{2} \dot{\beta} \cos \beta \sin \varphi_{2} \\
& \left.-l_{3} \dot{\varphi}_{3} \sin \gamma \sin \varphi_{3}+l_{3} \dot{\gamma} \cos \gamma \sin \varphi_{3}\right) \hat{k}
\end{aligned}
$$

The modeling of the acceleration for the point $\mathrm{P}_{1}$ :

$$
\begin{aligned}
& \overrightarrow{r_{1}}=\left[l_{1} \dot{\alpha}^{2} \cos \alpha+l_{1} \dot{\alpha} \sin \alpha\right] \hat{\imath} \\
& +[-\sin \\
& \propto \sin \varphi_{1}\left(l_{1} \dot{\varphi}_{1}+l_{1} \dot{\alpha}^{2}\right) \\
& +2\left(l_{1} \dot{\alpha} \dot{\varphi}_{1} \cos \propto \cos \varphi_{1}\right) \\
& \left.+l_{1}\left(\ddot{\varphi}_{1} \sin \propto \cos \varphi_{1}+\ddot{\propto} \cos \propto \sin \varphi_{1}\right)\right] \hat{\jmath} \\
& +[-\sin \\
& \propto \cos \varphi_{1}\left(l_{1} \varphi_{1}^{2}+l_{1} \propto^{2}\right) \\
& -2\left(l_{1} \dot{\alpha} \dot{\varphi}_{1} \cos \propto \sin \varphi_{1}\right)+l_{1}\left(-\ddot{\varphi}_{1} \sin \right. \\
& \left.\left.\propto \sin \varphi_{1}+\ddot{\propto} \cos \propto \cos \varphi_{1}\right)\right] \hat{k} \\
& \overrightarrow{\ddot{r}_{2}}=\left[l_{2} \dot{\beta}^{2} \cos \beta+l_{2} \ddot{\beta} \sin \beta+l_{1} \dot{\alpha}^{2} \cos \alpha+l_{1} \dot{\alpha} \sin \alpha\right] \hat{\imath} \\
& +\left[-l_{2} \sin \beta \sin \varphi_{2}\left(\dot{\varphi}_{2}{ }^{2}+\dot{\beta}^{2}\right)\right. \\
& +2\left(l_{2} \dot{\beta} \dot{\varphi}_{2} \cos \beta \cos \varphi_{2}\right) \\
& +l_{2}\left(\ddot{\varphi}_{2} \sin \beta \cos \varphi_{2}+\ddot{\beta} \cos \beta \sin \varphi_{2}\right) \\
& -\sin \\
& \propto \sin \varphi_{1}\left(l_{1} \dot{\varphi}_{1}+l_{1} \dot{\alpha}^{2}\right) \\
& +2\left(l_{1} \dot{\alpha} \dot{\varphi}_{1} \cos \propto \cos \varphi_{1}\right) \\
& \left.+l_{1}\left(\ddot{\varphi}_{1} \sin \propto \cos \varphi_{1}+\ddot{\propto} \cos \propto \sin \varphi_{1}\right)\right] \hat{\jmath} \\
& +\left[-l_{2} \sin \beta \cos \varphi_{2}\left({\dot{\varphi_{2}}}^{2}+\dot{\beta}^{2}\right)\right. \\
& -2\left(l_{2} \dot{\beta} \dot{\varphi}_{2} \cos \beta \sin \varphi_{2}\right) \\
& \left.+l_{2}\left(-\varphi_{2} \sin \beta \sin \varphi_{2}+\dot{\beta} \cos \beta \cos \varphi_{2}\right)\right] \hat{k} \\
& \overrightarrow{\vec{r}_{3}}=\left[l_{3} \dot{\gamma}^{2} \cos \gamma+l_{3} \ddot{\gamma} \sin \gamma\right] \hat{\imath} \\
& +\left[-l_{3} \sin \gamma \sin \varphi_{3}\left({\dot{\varphi_{3}}}^{2}+\dot{\gamma}^{2}\right)\right. \\
& +2\left(l_{3} \dot{\gamma} \dot{\varphi}_{3} \cos \gamma \cos \varphi_{3}\right) \\
& \left.+l_{3}\left(\ddot{\varphi}_{3} \sin \gamma \cos \varphi_{3}+\ddot{\gamma} \cos \gamma \cos \varphi_{3}\right)\right] \hat{\jmath} \\
& +\left[-l_{3}\left(-\ddot{\varphi}_{3} \sin \gamma \sin \varphi_{3}+\ddot{\gamma} \cos \gamma \cos \varphi_{3}\right] \hat{k}\right.
\end{aligned}
$$

To perform the simulation of the human gait taking into account the mathematical modeling, it is proceeded to obtain the position data in the time of the segments of the leg with the Tracker ${ }^{\circledR}$ software. In this program the body is tracked from the trunk to the feet taking mass points and assigning the mass value of each segment respectively, according to the book Model of the human bipedal gait using Modelica ${ }^{\circledR}$ and taking as reference the average person value.

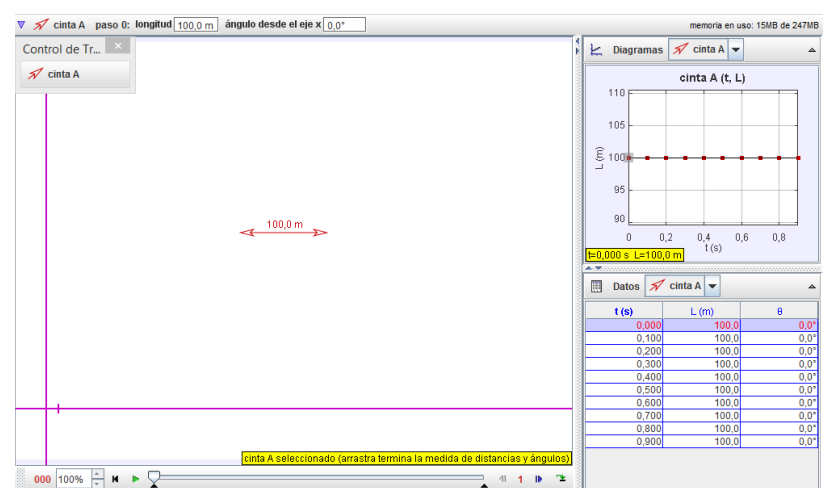

Fig. 9. Assignment of plane dimensions in Tracker. 
The values shown in the lower left part of the Figure 9 are taken from the human gait to stablish the datapoints, and after that it is generated an Excel file where the file is subsequently imported into the MATLAB database. In MATLAB, dynamic programming is carried out which consists of joining all the specific masses of the program to the shape of the human body, taking them from the data thrown by Tracker ${ }^{\circledR}$. The displacement of each section linked to another is implemented taking into account the position and speed values by assigning time values of each axe (x, y) and section (hips, knees, ankles). To be able to analyze the point-to-point image, as shown in fig. 10 .

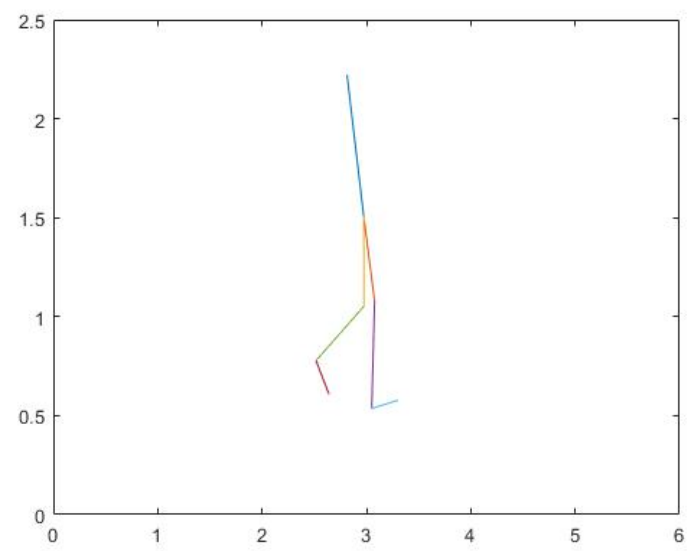

Fig. 10. Result of the simulation. Representation of the segments that allows the movement in the gait.

Once the reference frame for the representation of the program is stablished, that is the data of the work, a dynamic graph is made up such that once each iteration is finished, it deletes the content of the window and responds to the new position of the segments: Resulting in the simulation from the MATLAB software, in which the human displacement can be clearly analyzed segment by segment. For the development of this simulation it is necessary to consider the center of mass of Fig. 9; in this case is around the hip (which is the point of stabilization of movement).

Likewise, it is very important to define the reference frame in the Tracker ${ }^{\circledR}$ program in order to obtain adequate dimensional accuracy, taking into account that all the centers of mass are in a positive position on both the $\mathrm{x}$ axis and the $\mathrm{y}$ axis. When the trajectory is generated in MATLAB, it is necessary that each movement bound to disappear at the moment of the simulation, because if it is not, the trace will be marked while it is running. That is, if the memory of the drawing is not erased at the end of each iteration, it is obtained a graph like the one in fig 11 .

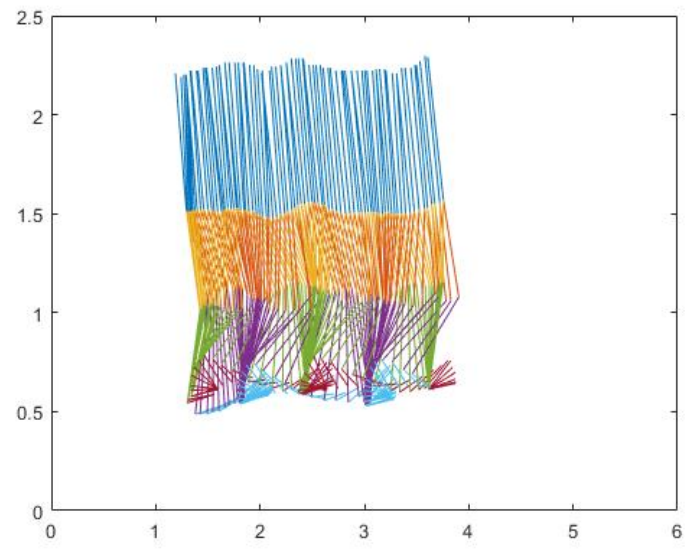

Fig. 11. Result of simulation with memory.

\section{Conclusions}

According to the results and the tests carried out in this work, it is possible to identify the movement generated for the lower train for the gait requires a synchronization in the advance of each joint, an agreement with the vectorially assigned position in the database.

The above, in order to avoid lags in the movement, since this could generate an alteration in the functioning of the physical assembly required for the application of the movement initially discussed in here. That is, the lengths of the segments would be variable and therefore the rigid structure would be unstable.

Given the previously proposed, it was possible to analyze (after performing different data assignment tests), that in Tracker ${ }^{\circledR}$ the tracking points should be taken in the same time rate for each of the joints, since if not, for example taking the point of the ankle every 2 jumps, and for the hip every jump, the longitudinal variations present in the design would prevent a realistic visualization of the model. This would lead to undesirable traction and compression efforts in the extremities.

This mathematical modeling and simulation of the human gait allows to base the deepening for the realization of studies in the medical field and in engineering research as well. It is recommended for future modeling that this can continue to be studied and applied in 3D systems.

\section{Acknowledgement}

The authors are grateful to the Piloto de Colombia University and Nueva Granada Military University.

This is an Open Access article distributed under the terms of the Creative Commons Attribution License

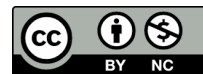

\section{References}

[1] J. Javier. Sánchez Lacuesta, Jaime. Prat, Javier. Sánchez-Lacuesta. biomecánica de la marcha humana normal y patológica. Instituto de Biomecánica de Valencia (España).

[2] Bravo, L. E. C., \& Garzón, M. A. R. (2005). Modelamiento de la marcha humana por medio de gráficos de unión. Tecnura, 8(16),34.

[3] Luengas, L. A., Marín, C. A., \& González, J. F. (2013). Modelo de la marcha bípeda humana usando Modelica. Visión electrónica, (2), 110-124.
[4] Knudson,D. Fundamentals of Biomechanics,Second edition.160240 .

[5] Rostami, M., \& Bessonnet, G. (1998, May). Impactless sagittal gait of a biped robot during the single support phase. In Robotics and Automation, 1998. Proceedings. 1998 IEEE International Conference on (Vol. 2, pp. 1385-1391). IEEE.

[6] Goswami, A., Espiau, B., \& Keramane, A. (1996, April). Limit cycles and their stability in a passive bipedal gait. In Robotics and 
Xue M. González, Diego F. Valero, Oscar Avilés, Ruben D. Hernández and Jair L. Loaiza/

\section{Journal of Engineering Science and Technology Review 13 (1) (2020) 18 - 25}

Automation, 1996. Proceedings., 1996 IEEE International Conference on (Vol. 1, pp. 246-251). IEEE.

[7] Crompton, R. H., Weijie, L. Y. W., Günther, M., \& Savage, R. (1998). The mechanical effectiveness of erect and "bent-hip, bentknee" bipedal walking in Australopithecus afarensis. Journal of Human Evolution, 35(1), 55-74.

[8] Ardila, J. C. C., \& Escarpeta, J. M. R. (2012). Modelo matemático y herramienta de simulación de exoesqueleto activo de cinco segmentos. Revista Guillermo de Ockham, 10(2).

[9] Cifuentes, C., Martínez, F., \& Romero, E. (2010). Análisis teórico y computacional de la marcha normal y patológica: una revisión. Revista Med, 18(2), 182-196.

[10]P. Giovanni Sánchez and C. Lely Adriana Luengas, "Aplicación del modelo RC en sistemas biológicos (mecánica ventilatoria)," Revista Inventum, vol. 6, (10), pp. 16-23, 2011. Available: http://ezproxy.unipiloto.edu.co/docview/2018733465? accountid=5 0440 .

DOI: http://dx.doi.org/10.26620/uniminuto.inventum.6.10.2011.16-23.

[11]E. P. Flores et al, "Diseño del mecanismo actuador de un dedo robot antropomórfico/Design of the drive mechanism for an anthropomorphic robotic finger," Revista Facultad De Ingeniería Universidad De Antioquia, (58), pp. 153-162, 2011. Available: http://ezproxy.unipiloto.edu.co/docview/1612426604? accountid=5 0440.

[12]É. A. P. Flores et al, "ANÁLISIS CINEMÁTICO Y DISEÑO DE UN MECANISMO DE CUATRO BARRAS PARA FALANGE PROXIMAL DE DEDO ANTROPOMÓRFICO/KYNEMATI C ANALYSIS AND DESIGN OF A FOUR-BAR MECHANISM FOR PROXIMAL FINGER PHALANX OF ANTHROPOMORPHIC FINGER," Ciencia e Ingeniería Neogranadina, vol. 20, (1), pp. 45-59, 2010. Available: http://ezproxy.unipiloto.edu.co/docview/752130812?accountid $=50$ 440.

[13]F. Muri et al, "Diseño de un sistema de rehabilitación para miembro superior en entorno de realidad virtual/DESIGN OF A UPPER LIMB REHABILITATION SYSTEM IN VIRTUAL REALITY ENVIRONMENT/PROJETO DO SISTEMA DE REABILITAÇÃO DO MEMBRO SUPERIOR EM AMBIENTE DE REALIDADE VIRTUAL," Revista Ingeniería Biomédica, vol. 7, (14), pp. 81-89, 2013. Available: http://ezproxy.unipiloto.edu.co/docview/1497034704?accountid=5 0440 .

[14]M. A. P. Romero et al, "Prototipo de mano robótica antropométrica sub-actuada/Sub-actuated anthropometric robotic prototype hand," Revista Facultad De Ingeniería Universidad De Antioquia, (65), pp. 46-59, $2012 . \quad$ Available: http://ezproxy.unipiloto.edu.co/docview/1614170607?accountid=5 0440 .

[15]Fredy Hernán Martínez Sarmiento, J. G. Edwar and D. A. Zárate Díaz, "Concepto de robot humanoide antropométrico para investigación en control," Tecnura, vol. 19, pp. 55-65, 2015. Available:

http://ezproxy.unipiloto.edu.co/docview/1865308025?accountid=5 0440 . DOI: http://dx.doi.org/10.14483/udistrital.jour.tecnura.2015.SE1.a04.

[16]B. T. Amador et al, "Metodologia para dimensionamiento de mecanismo policéntrico de rodilla utilizando análisis de marcha y algoritmos genéticos," Revista Ingeniería Biomédica, vol. 6, (11), pp. $30-45, \quad 2012 . \quad$ Available: http://ezproxy.unipiloto.edu.co/docview/1399140885?accountid=5 0440 . 